\section{LA PERSPECTIVA DEL GÉNERO EN EL CÁNCER: UNA VISIÓN RELEVANTE Y NECESARIA}

\author{
Josep M. - Borràs \\ Universidad de Barcelona \\ jmborras@iconcologia.net
}

\section{GENDER PERSPECTIVE IN CANCER: AN IMPORTANT AND NECESSARY VISION}

Cómo citar este artículo/Citation: Borràs, J. M. (2015). "La perspectiva del género en el cáncer: una visión relevante y necesaria". Arbor, 191 (773): a231. doi: http://dx.doi. org/10.3989/arbor.2015.773n3001
Copyright: @ 2015 CSIC. Este es un artículo de acceso abierto distribuido bajo los términos de la licencia Creative Commons Attribution-Non Commercial (by-nc) Spain 3.0.

Recibido: 9 diciembre 2014. Aceptado: 24 abril 2015.

RESUMEN: La perspectiva del género en el cáncer ha formado parte de la gran mayoría de análisis del impacto de esta enfermedad, dada su indudable relevancia. En este artículo, exploraremos brevemente tres tipos de datos que avalan esta afirmación, la incidencia y mortalidad diferencial según género, la supervivencia observada y el comportamiento respecto a los factores de riesgo, como el tabaco. Finalmente, comentaremos sus posibles consecuencias para la estrategia de control del cáncer en nuestro país. Cualquier objetivo de control del cáncer debe ser planteado teniendo en cuenta la relevancia de la perspectiva del género.

PALABRAS CLAVE: estrategia de control; género; tabaco.
ABSTRACT: The gender perspective has been part of most analyses of the impact of cancer, given its obvious importance. In this article, we will briefly explore three types of data supporting this statement, the incidence and differential mortality according to gender, the survival rates observed and behavior with regard to risk factors such as tobacco. Lastly, we will discuss their possible consequences for Spain's cancer control strategy. Any cancer control target proposed must consider the importance of the gender perspective.

KEYWORDS: control strategy; gender; tobacco 


\section{EL IMPACTO DEL CÁNCER SEGÚN GÉNERO: ALGU- NOS DATOS}

La incidencia de cáncer presenta aspectos propios para cada género y que tradicionalmente han motivado tanto análisis diferenciados de forma sistemática en todas las publicaciones sobre el tema (Ferlay et al., 2013), como la necesidad de evaluar las tendencias de cada tumor con análisis específicos según género y edad y la frecuencia de los distintos tipos de cáncer según el género. Así, en nuestro país y con los datos de las estimaciones realizadas en el marco del Observatorio Europeo del Cáncer para el año 2012 (los métodos y resultados de las estimaciones para Europa se pueden revisar en la web del observatorio (http:// www.eco.iarc.fr) (Ferlay et al., 2013) se puede constatar una mayor incidencia de casos de cáncer en los hombres que en las mujeres, aunque los tumores más frecuentes son claramente distintos. Así, cerca de un tercio de tumores en las mujeres son diagnosticados como cáncer de mama, mientras que en los hombres el predominio del cáncer más frecuente es menor, dado que el $22 \%$ son atribuibles al cáncer de próstata, seguido por el de pulmón y colorrectal.

Los tumores relacionados con el tabaco (laringe, cavidad oral, esófago, pulmón, vejiga urinaria, páncreas y riñón como los más relevantes) explican cerca de la mitad de los cánceres en los hombres por solo una quinta parte en las mujeres. Por su parte, los tumores ginecológicos, incluyendo el cáncer de mama, explican el $40 \%$ de todos los tumores de la mujer. Para tener una visión del contexto europeo, cabe mencionar que el dato más diferenciado es la creciente importancia del cáncer de pulmón en las mujeres, aunque su frecuencia es todavía baja, al partir de unas cifras muy bajas en prevalencia del tabaquismo en los años sesenta y setenta. Sin embargo, esta tendencia ha cambiado y actualmente, la prevalencia de fumadores jóvenes es similar en ambos sexos (Fernandez et al., 2003). Cabe mencionar que dos tumores relacionados con el tabaco, el cáncer de laringe y el cáncer de vejiga urinaria son los que tienen mayor diferencial de incidencia y mortalidad según género en nuestro país, siguiendo un patrón similar al de otros países europeos y que puede estar relacionado con el tipo de tabaco fumado y con los otros factores de riesgo que se asocian a estos tumores (exposición al consumo de alcohol y carcinógenos ambientales y laborales, respectivamente).

La tendencia del cáncer de pulmón en mujeres ha requerido una evaluación detallada tanto en incidencia como en mortalidad por grupos de edad para evaluar los indicios iniciales de cambio de tendencia hacia el incremento (Borras et al., 2001; Izarzugaza et al., 2010) , y traducirlo en las prioridades de intervención preventiva, desde el mismo inicio de la Estrategia en cáncer en 2005 (Borras et al., 2010) . En este sentido, es muy relevante la contribución realizada a estos análisis por los registros de cáncer de base poblacional existentes en España, que han permitido un análisis conjunto de los datos de incidencia y mortalidad, lo cual facilita disponer de un análisis adaptado a nuestra realidad epidemiológica. Sin embargo, sería necesario extender la cobertura de los registros poblacionales a un mayor territorio y porcentaje de población con objeto de representar más fielmente la situación española.

\section{LA SUPERVIVENCIA DEL CÁNCER: LA RELEVANCIA DEL GÉNERO}

Un segundo ámbito en el que la perspectiva de género es fundamental es la explicación del diferencial de supervivencia en cáncer. Es conocido que las mujeres presentan una mayor supervivencia conjuntamente para todos los cánceres. Para evaluarlo, disponemos de los datos de EUROCARE, que analizan la supervivencia relativa (ajustada por la probabilidad de morir por otras causas de muerte) a los 5 años de los pacientes diagnosticados en el período 2000-2007 en los registros poblacionales europeos (De Angelis et al., 2014). Los datos muestran el progreso realizado en diferentes tumores para todas las regiones europeas, así como ofrecen la posibilidad de comparar los datos de nuestro país con el resto de países europeos y, por lo tanto, identificar los márgenes de mejora que podemos tener en nuestro sistema sanitario.

La supervivencia de las mujeres es superior a la de los hombres porque los tumores más frecuentes en ellas presentan mejores supervivencias; si bien es cierto que a ello contribuyen los programas de diagnóstico precoz instaurados desde hace años para detectar la patología mamaria. Así, por ejemplo, el tumor más frecuente en mujeres es el cáncer de mama con una supervivencia relativa a los 5 años del $82 \%$ para Europa mientras que en España es del 83,8\%. Mientras que en próstata es del $84,4 \%$ en Europa y del $84,7 \%$ en España, y en el cáncer de pulmón la supervivencia es del $13 \%$ en Europa y del $10 \%$ en España. Un dato relevante es que la supervivencia del cáncer de pulmón es discretamente superior en las mujeres que en los hombres (15,9\% para las mujeres europeas y $12 \%$ para los hombres). Esta diferencia se debe al mejor pronóstico que tienen los adenocarcinomas en 
relación a los tumores escamosos, histología mucho más frecuente en hombres. Otros tumores en los que hay una discreta mejor supervivencia en mujeres que en hombres son el cáncer de recto ( 57,3 vs $54,9 \%$ ) o estómago ( 27,7 vs $23,7 \%)$, melanoma ( 86,6 vs $79,2 \%$ ), pero no sucede así en el de colon. Las razones detrás de estas diferencias pueden ser muy variadas, entre las que vale la pena mencionar diferentes tipos histológicos como en el caso del cáncer de pulmón, mientras que en estos tumores también puede ser debido a un menor retraso diagnóstico o una menor comorbilidad. Los datos permiten explicar que la mejor supervivencia del cáncer en las mujeres se debe evaluar por un conjunto de factores, el más importante entre los cuales es el mejor pronóstico de los tumores más frecuentes en las mujeres.

Otro aspecto relevante de estos datos tiene que ver con el diferencial de supervivencia de nuestro país en relación a los países con mejores datos de supervivencia en Europa. Ciertamente, nos hemos situado siempre por encima de la media europea en supervivencia para todos los tumores, pero es obvio que todavía tenemos un camino que recorrer en nuestros resultados clínicos si nos comparamos con los países que tienen las cifras de supervivencia más elevadas y esto sucede en todos los tumores. Aunque no debemos olvidar los problemas metodológicos derivados de comparar resultados entre países, debemos analizar cómo podemos mejorar nuestra supervivencia focalizándonos en aspectos como mejorar el acceso al diagnóstico, evitando retrasos innecesarios, y en la calidad de la atención oncológica. Un paso ineludible para esto es disponer de más y mejores datos de los resultados clínicos, ámbito en el que nuestras carencias son significativas. Sin embargo, no debemos olvidar que los resultados de supervivencia se correlacionan fuertemente con el nivel socioeconómico del país (De Angelis et al., 2014), lo cual es indicativo de que nuestro esfuerzo se debe centrar en mejorar la ordenación de nuestra actividad asistencial y en la evaluación de la misma, concentrando algunos tratamientos de alta complejidad, por ejemplo. El desafío en este ámbito es claro y la perspectiva de género puede ayudar a analizar con más detalle las causas de las diferencias en supervivencia y así disponer de elementos relevantes para avanzar en la mejora de los resultados clínicos.

\section{LOS RETOS DE LA PREVENCIÓN DEL TABAQUISMO: PREVENIR LA EPIDEMIA DE CÁNCER DE PULMÓN EN MUJERES}

Un tercer ámbito en el cual la perspectiva de género es muy relevante es el análisis de los factores de riesgo relacionados con el cáncer. Nos centraremos en el tabaquismo como factor más reconocido e investigado por su fuerte, consistente y establecida asociación con numerosos tipos de cáncer, aunque otros factores como la obesidad y el sobrepeso o la exposición solar intensa también tienen aspectos de género muy relevantes. Como hemos comentado previamente, un aspecto que durante muchos años ha definido la incidencia del cáncer en nuestro país ha sido la muy baja frecuencia de los tumores relacionados con el tabaco y singularmente, el cáncer de pulmón al ser el que ha mostrado un mayor riesgo atribuible, en torno al $80 \%$ de estos cánceres se asocian al tabaquismo.

Desde hace algunos años, se han reconstruido las cohortes históricas de inicio del consumo de tabaco en nuestro país y se ha podido observar que las mujeres no empezaron a fumar de forma importante hasta los años setenta y solo en las cohortes jóvenes; y que la epidemia del tabaquismo empezó en las mujeres de nivel socio-económico más elevado para posteriormente difundirse entre las de nivel bajo, mientras que en los años ochenta el proceso de abandonar el hábito tabáquico se inició también en las de nivel socio-económico más elevado (Borras et al., 2000). En este sentido, la diseminación de la epidemia del tabaquismo siguió un patrón de difusión de la innovación muy marcado. La epidemia del tabaquismo se sigue habitualmente de la epidemia del cáncer de pulmón al cabo de 35-40 años, lo cual estamos comprobando actualmente en nuestro caso, tal y como era previsible (Bilal et al., 2014). La prevalencia del consumo de tabaco entre los jóvenes es similar en ambos sexos desde los inicios de este siglo, según demuestran las encuestas nacionales de salud, lo cual no es una buena noticia ya que se debe a que el descenso ha sido muy notable en los hombres pero no tanto en las mujeres (Tabla 1 ). Los esfuerzos de prevención deben ser diferenciales según género, porque las motivaciones para fumar y para dejar de hacerlo pueden ser también distintas (Dixon y Banwell, 2009). Un elemento clave es el retraso en la edad de inicio para fumar y el adelanto de la edad de abandono del tabaquismo y este debe ser un objetivo crucial de las estrategias preventivas del consumo de tabaco en nuestro país. Los progresos son manifiestos, pero queda mucho por hacer en este ámbito clave de la prevención del cáncer y de múltiples enfermedades crónicas. Los datos de la Tabla 1 y de la Figura 1 muestran gráficamente el progreso y el reto de continuar el mismo en los próximos años. 
Tabla 1. Tasa de fumadores en la población española de 15 a 24 años (1993-2011), distribución por sexo [http:// pestadistico.inteligenciadegestion.msssi.es/ArbolNodos.aspx]

\begin{tabular}{llllllll}
\hline & $\mathbf{1 9 9 3}$ & $\mathbf{1 9 9 5}$ & $\mathbf{1 9 9 7}$ & $\mathbf{2 0 0 1}$ & $\mathbf{2 0 0 3}$ & $\mathbf{2 0 0 6}$ & $\mathbf{2 0 1 1}$ \\
\hline Varones & 38,60 & 38,95 & 35,95 & 36,48 & 34,79 & 24,96 & 22,50 \\
\hline Mujeres & 33,83 & 40,54 & 34,48 & 36,92 & 31,10 & 28,93 & 22,50 \\
\hline
\end{tabular}

Figura 1. Tendencia de la tasa de población fumadora en los mayores de 15 años (1993-2011), distribución por sexo [http://pestadistico.inteligenciadegestion.msssi.es/ArbolNodos.aspx]

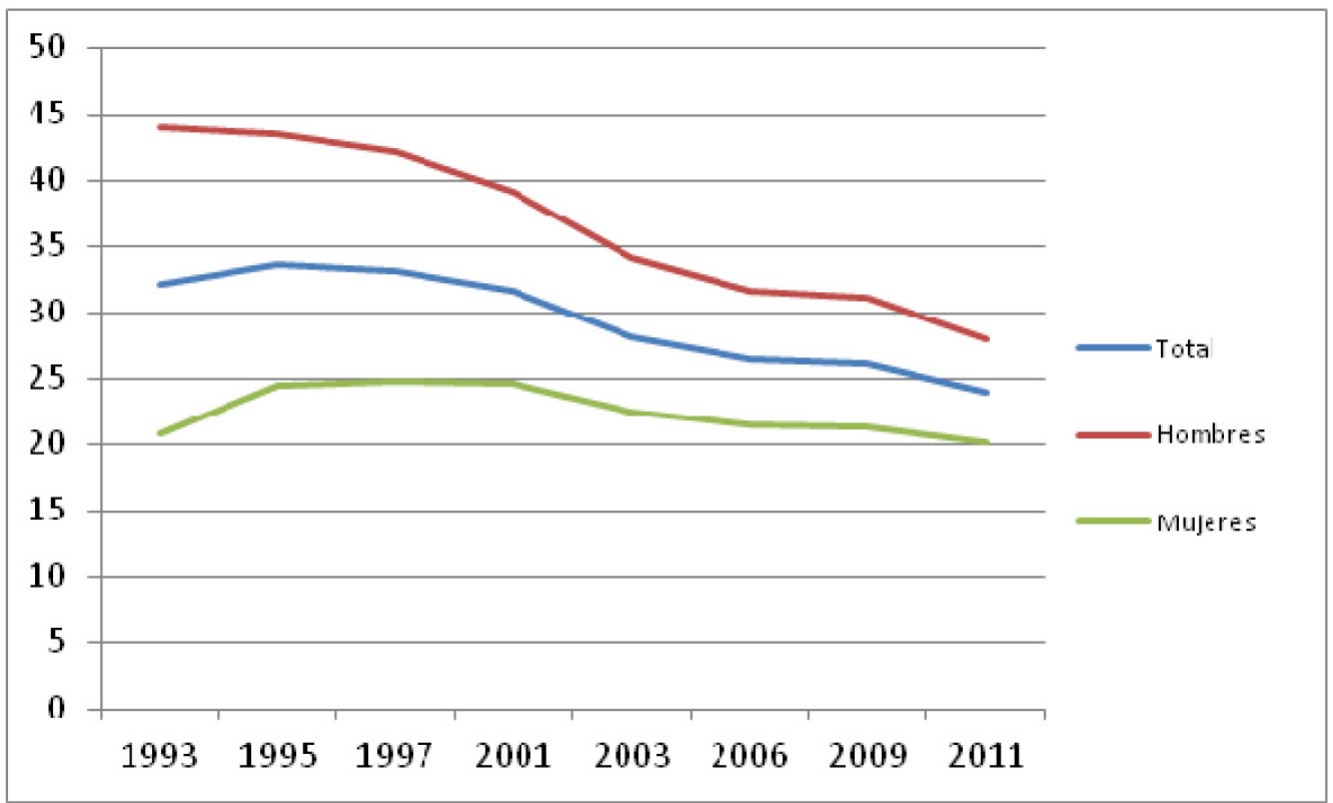

\section{LOS RETOS DE LA ESTRATEGIA EN CÁNCER DEL SIS- TEMA NACIONAL DE SALUD EN EL MARCO DEL ENFO- QUE DE GÉNERO}

La necesidad del enfoque del género en el marco de la Estrategia en cáncer del Sistema Nacional de Salud ha sido asumida como un reto en los objetivos definidos desde su inicio en el 2005. De este modo, los objetivos de la Estrategia de eprevención de los factores de riesgo o de cribado poblacional han sido definidos específicamente para cada género, adaptándose a las diferentes prevalencias y a sus tendencias en el tabaquismo o en la obesidad (VV. AA., 2010). Las prioridades del control del cáncer definidos en la Estrategia se centran en distintos ámbitos: prevención primaria, cribado poblacional, atención sanitaria a adultos, oncología pediatría, atención psicosocial, cuidados paliativos e investigación. Además, se incluye un ámbito de sistemas de información con los indicadores de evaluación de la propia estrategia.
Los principales objetivos en el ámbito preventivo son compartidos con otras estrategias al tener en común muchos factores de riesgo con patologías crónicas. Entre estos, podemos destacar el tabaquismo y el sobrepeso, sobretodo en edades jóvenes, la prevención de carcinógenos ambientales y laborales así como la prevención del consumo excesivo de alcohol. Un objetivo específico del cáncer es la prevención de la exposición excesiva al sol y la vacunación sobre la hepatitis B y el virus del papiloma humano. Los objetivos de cribado se centran en el de cáncer de mama bienal entre 50 y 69 años mediante mamografía, la detección precoz del cáncer de cuello uterino mediante citología, aunque evidencia reciente también permite utilizar la detección del virus del papiloma humano en la citología; y la de cáncer colorrectal mediante detección de sangre oculta en las heces bienalmente entre 50 y 69 años. Todos estos objetivos se han acordado específicos según el género, lo 
cual remarca la importancia de este enfoque en el planteamiento de la propia Estrategia. Este hecho es más difícil de individualizar en el ámbito de la atención sanitaria, donde los objetivos se centran en aspectos organizativos como la necesidad de mejorar el proceso diagnóstico, la necesidad de promover y consolidar la atención multidisciplinaria y la de centralizar tratamientos de alta complejidad y baja frecuencia. Otros aspectos singulares que se pueden destacar son la necesidad de potenciar la atención psicosocial y pediátrica especializada.

Un aspecto clave que debemos potenciar en nuestro país es la necesidad de disponer de mejores datos de evaluación de resultados clínicos que permitan conocer los aspectos que debemos mejorar. Junto a estos resultados, también necesitamos registros de cáncer de base poblacional, de los que actualmente solo disponemos de información de solo una parte de la población española. Deberíamos avanzar en una mayor cobertura poblacional progresiva así como en aumentar las variables relacionadas con el estadio en el momento del diagnóstico, la comorbilidad del paciente y en el tipo de tratamiento recibido.

En síntesis, el impacto del cáncer en la sociedad solo puede ser entendido de forma racional teniendo siempre presente la perspectiva del género, tanto cuando analizamos la incidencia, la supervivencia o los factores de riesgo asociados al cáncer. Este hecho supone que cualquier objetivo de control del cáncer debe ser planteado teniendo en cuenta la relevancia de la perspectiva del género, como desde su inicio se ha tratado de llevar a cabo en la Estrategia en cáncer junto con las guías europeas de control de cáncer (Abreht, Borras y Conroy, 2014; Martin-Moreno, Albreht y Rados-Krnel, 2013).

\section{BIBLIOGRAFÍA}

Abreht, T., Borras, J. y Conroy, F. (2014). European guide for quality cancer control programmes. Ljubjliana: European partnership action against cancer.

Bilal, U., Fernandez, E., Beltran, P., NavasAcien, A., Bolumar, F. y Franco, M. (2014). Validation of a method for reconstructing historical rates of smoking prevalence. American Journal of Epidemiology, 179, 1, pp. 15-19. DOI: kwt224 [pii]10.1093/aje/kwt224.

Borras, J., Borras, J. M., Galceran, J., Sanchez, V., Moreno, V. y Gonzalez, J. R. (2001). Trends in smoking-related cancer incidence in Tarragona, Spain, 1980-96. Cancer Causes Control, 12, 10, pp. 903-908.

Borras, J. M., Colomer, C, Soria, P., Lopez, R. (2010). Priorities for cancer control in Spain. Annals of Oncology, 21 Suppl 3, pp. iii111-114. DOI: mdq099 [pii]10.1093/annonc/mdq099.

Borras, J. M., Fernandez, E., Schiaffino, A., Borrell, C. y La Vecchia, C. (2000). Pattern of smoking initiation in Catalonia, Spain, from 1948 to 1992. American Journal of Public Health, 90, 9, pp. 1459-1462.
De Angelis, R., Sant, M., Coleman, M. P., Francisci, S., Baili, P., Pierannunzio, D. et al. (2014). Cancer survival in Europe 1999-2007 by country and age: results of EUROCARE--5-a population-based study. The Lancet Oncology, 15, 1, pp. 23-34. DOI: S1470-2045(13)70546-1 [pii]10.1016/S1470-2045(13)70546-1.

Dixon, J. y Banwell, C. (2009). Theory driven research designs for explaining behavioural health risk transitions: the case of smoking. Social Science \& Medicine, 68,12 , pp. 2206-2214. DOI: S02779536(09)00186-5 [pii]10.1016/j.socscimed.2009.03.025.

Ferlay, J., Steliarova-Foucher, E., LortetTieulent, J., Rosso, S., Coebergh, J. W., Comber, H. et al. (2013). Cancer incidence and mortality patterns in Europe: estimates for 40 countries in 2012. European Journal of Cancer, 49, 6, pp. 1374-1403. DOI: S0959-8049(13)000075 [pii]10.1016/j.ejca.2012.12.027.

Fernandez, E., Schiaffino, A., Garcia, M., Salto, E., Villalbi, J. R. y Borras, J. M. (2003). Prevalencia del consumo de tabaco en España entre 1945 y 1995. Reconstrucción a partir de las Encuestas Nacionale de Salud [Smoking in Spain, 1945-1995. A retrospective analysis based on the Spanish National Health Interview Surveys]. Medicina Clínica, 120, 1, pp. 14-16. DOI: 13042014 [pii].

Izarzugaza, M. I., Ardanaz, E., Chirlaque, M. D., Font, C., Ameijide, A. y Linares, C. (2010). Tobacco-related tumours of the lung, bladder and larynx: changes in Spain. Annals of Oncology, 21 Suppl 3, pp. iii52-60. DOI: mdq084 [pii]10.1093/ annonc/mdq084.

Martin-Moreno, J., Albreht, T. y RadosKrnel, S. (eds.) (2013) Boosting innovation and cooperation in european cancer control. Ljubljana: National Institute of Health of the Republic of Slovenia \& World Health Organization on behalf of the European Observatory on Health Systems and Policies.

VV. AA. (2010). Estrategia en cáncer del Sistema Nacional de Salud. Madrid: Ministerio de Sanidad y Política Social. 